\title{
Gesetz der Russländischen Föderation zur Änderung der Verfassung der Russländischen Föderation vom 14. März 2020 Nr. 1-FKZ „Über die Verbesserung der Regelung einzelner Fragen der Organisation und Arbeitsweise der öffentlichen Gewalt"
}

(Sobranie zakonodatel'stva Rossijskoj Federacii 2020 Nr. 11 Pos. 1416)

\section{Artikel 1}

Die durch Volksabstimmung am 12. Dezember 1993 angenommene Verfassung der Russländischen Föderation (Rossijskaja gazeta vom 25. Dezember 1993) wird wie folgt geändert:

$$
[\ldots]^{1}
$$

\section{Artikel 2}

1. Eine allrussische Abstimmung über die Billigung der in Artikel 1 dieses Gesetzes der Russländischen Föderation zur Änderung der Verfassung der Russländischen Föderation (im Folgenden jeweils: allrussische Abstimmung bzw. dieses Gesetz) wird nach Inkrafttreten dieses Gesetzes und unter der Voraussetzung, dass das Verfassungsgericht der Russländischen Föderation in Übereinstimmung mit Artikel 3 dieses Gesetzes ein Gutachten über die Vereinbarkeit der nicht in Kraft getretenen Vorschriften dieses Gesetzes mit den Vorschriften der Kapitel 1, 2 und 9 der Verfassung der Russländischen Föderation sowie der Regelung über das Inkrafttreten von Artikel 1 dieses Gesetzes mit der Verfassung der Russländischen Föderation erstattet.

2. Das Recht zur Initiierung der allrussischen Abstimmung steht dem Präsidenten der Russländischen Föderation zu. Der Präsident der Russländischen Föderation setzt die allrussische Abstimmung durch Ukaz an.

3. Der Ukaz des Präsidenten der Russländischen Föderation, mit dem die allrussische Abstimmung angesetzt wird, enthält die Frage, die Gegenstand der allrussischen Abstimmung ist, und bestimmt den Tag der allrussischen Abstimmung in Übereinstimmung mit Absatz 5 dieses Artikels.

4. Der Ukaz des Präsidenten der Russländischen Föderation, mit dem die allrussische Abstimmung angesetzt wird, ist spätestens am Tag nach seiner Unterzeichnung offiziell bekanntzumachen.

1 Die Änderungen sind im vorstehend abgedruckten Text der Verfassung der Russländischen Föderation berücksichtigt und werden daher hier nicht wiedergegeben. 
5. Um möglichst günstige Bedingungen für die Teilnahme der Bürger der Russländischen Föderation an der allrussischen Abstimmung sicherzustellen, darf die allrussische Abstimmung frühestens 30 Tage nach dem Tag der offiziellen Bekanntmachung des Ukaz des Präsidenten der Russländischen Föderation, mit dem die allrussische Abstimmung angesetzt wird, stattfinden.

Wenn bestimmt wird, dass die allrussische Abstimmung an einem Arbeitstag stattfindet, so ist dieser Tag kraft dieses Gesetzes ein arbeitsfreier Tag. Die Entlohnung der Arbeitnehmer an diesem Tag erfolgt in Übereinstimmung mit den Vorschriften des Arbeitsgesetzbuchs der Russländischen Föderation über die Entlohnung an arbeitsfreien Tagen (Feiertagen).

6. An der allrussischen Abstimmung können Bürger der Russländischen Föderation teilnehmen, die am Tag der allrussischen Abstimmung das Alter von 18 Jahren erreicht haben; ausgenommen sind Bürger, die gerichtlich entmündigt sind oder aufgrund eines Gerichtsurteils in Haftanstalten einsitzen.

7. Die Teilnahme der Bürger der Russländischen Föderation an der allrussischen Abstimmung erfolgt nach den Grundsätzen der allgemeinen gleichen und unmittelbaren Willensäußerung bei geheimer Abstimmung.

8. Die Teilnahme eines Bürgers der Russländischen Föderation an der allrussischen Abstimmung ist frei und freiwillig.

Niemand darf auf einen Bürger der Russländischen Föderation mit dem Ziel einwirken, ihn zu der Teilnahme oder Nichtteilnahme an der allrussischen Abstimmung zu nötigen oder ihn in seiner freien Willensäußerung zu behindern.

9. Die Vorbereitung und Durchführung der allrussischen Abstimmung erfolgen offen und transparent.

10. Folgende Stellen bereiten die allrussische Abstimmung vor und führen sie durch:

1) die Zentrale Wahlkommission der Russländischen Föderation (als die die allrussische Abstimmung vorbereitende und ihre Durchführung sicherstellende Wahlkommission);

2) die Wahlkommissionen der Subjekte der Russländischen Föderation;

3) die Wahlkommissionen der Bezirke;

4) die Wahlkommissionen an den Wahllokalen.

11. Die Zentrale Wahlkommission der Russländischen Föderation und die sonstigen Wahlkommissionen gehen bei der Vorbereitung und Durchführung der allrussischen Abstimmung von dem Erfordernis aus, die größtmöglichen Annehmlichkeiten für die an der allrussischen Abstimmungen Teilnehmenden zu schaffen.

12. Die Entscheidungen, die übergeordnete Wahlkommissionen in Fragen der Vorbereitung und Durchführung der allrussischen Abstimmung im Rahmen ihrer Zuständigkeit getroffen haben, sind für die untergeordneten Wahlkommissionen verbindlich.

13. Die Entscheidungen, die die Zentrale Wahlkommission der Russländischen Föderation und andere Wahlkommissionen in Fragen der Vorbereitung und Durchführung der allrussischen Abstimmung im Rahmen ihrer Zuständigkeit getroffen haben, sind für die föderalen Vollzugsorgane, die Vollzugsorgane der Subjekte der Russländischen Föderation, für die sonstigen staatlichen Organe, die Organe der örtlichen Selbstverwaltung, Organisationen, Amtsträger und Bürger der Russländischen Föderation verbindlich. 
14. Die Zentrale Wahlkommission der Russländischen Föderation stellt die Offenheit und Transparenz bei der Vorbereitung und Durchführung der allrussischen Abstimmung sicher, unter anderem schafft sie in Übereinstimmung mit Absatz 16 dieses Artikels die Bedingungen für die Beobachtung der Vorbereitung und Durchführung der allrussischen Abstimmung, der Feststellung der abgegebenen Stimmen und der Bestimmung der Ergebnisse.

15. Die Zentrale Wahlkommission der Russländischen Föderation:

1) bestätigt das Verfahren der allrussischen Abstimmung innerhalb dreier Tage ab dem Tag der offiziellen Bekanntmachung des Ukaz des Präsidenten der Russländischen Föderation, mit dem die allrussische Abstimmung angesetzt wird;

2) stellt sicher, dass die Bürger der Russländischen Föderation über die Vorbereitung und Durchführung der allrussischen Abstimmung informiert werden, insbesondere, dass sie die Möglichkeit erhalten, von dem Text dieses Gesetzes Kenntnis zu nehmen, und regelt das Verfahren der Akkreditierung von Vertretern der Massenmedien zur Teilnahme an der Berichterstattung über die Durchführung der allrussischen Abstimmung;

3) legt Form und den Text der Stimmzettel für die allrussische Abstimmung, das Verfahren der Herstellung und des Versands der Stimmzettel und das Verfahren der Überwachung von Herstellung und Versand fest;

4) stellt die bei der allrussischen Abstimmung abgegebenen Stimmen fest und bestimmt die Ergebnisse der allrussischen Abstimmung;

5) regelt die Finanzierung der Vorbereitung und Durchführung der allrussischen Abstimmung; dabei sind Regelungen für die Verwendung der für die Vorbereitung und Durchführung der allrussischen Abstimmung zur Verfügung gestellten Mittel, die Verteilung dieser Mittel, die Abrechnung und die Beschaffung von Waren, Arbeiten und Dienstleistungen im Zusammenhang mit der Vorbereitung und Durchführung der allrussischen Abstimmung durch die Wahlkommissionen zu treffen;

6) nimmt weitere Befugnisse im Bereich der Organisation der Vorbereitung und Durchführung der allrussischen Abstimmung wahr.

16. Zur Beobachtung der Abstimmung und der Auszählung der Stimmen der Teilnehmer der Abstimmung und der Feststellung der abgegebenen Stimmen ernennen die Gesellschaftskammer der Russländischen Föderation und die Gesellschaftskammern der Subjekte der Russländischen Föderation Beobachter.

Dabei kann die Gesellschaftskammer der Russländischen Föderation ab dem Tag der offiziellen Bekanntmachung des Ukaz des Präsidenten der Russländischen Föderation, mit dem die allrussische Abstimmung angesetzt wird, einen Beobachter in die Zentrale Wahlkommission der Russländischen Föderation entsenden, dem es unter anderem obliegt, die Vorbereitung der allrussischen Abstimmung zu beobachten. Die Gesellschaftskammer der Russländischen Föderation kann Beobachter auch in alle untergeordneten Wahlkommissionen entsenden. Die Gesellschaftskammern der Subjekte der Russländischen Föderation entsenden Beobachter in die auf dem Territorium des jeweiligen Subjekts der Russländischen Föderation gelegenen Wahlkommissionen. 
Die Gesellschaftskammer der Russländischen Föderation erlässt eine Regelung über die Entgegennahme von Vorschlägen für Kandidaturen für Beobachter und die Ernennung der Beobachter.

17. Die föderalen Vollzugsorgane, die Vollzugsorgane der Subjekte der Russländischen Föderation, die sonstigen staatlichen Organe, die Organe der örtlichen Selbstverwaltung, Organisationen und Amtspersonen sind verpflichtet, die Wahlkommissionen bei der Erfüllung ihrer Aufgaben im Bereich der Vorbereitung und Durchführung der allrussischen Abstimmung und bei der materiellen und technischen Sicherung der Vorbereitung und Durchführung der allrussischen Abstimmung (einschließlich der unentgeltlichen Bereitstellung erforderlicher Räumlichkeiten, Transportmittel, Kommunikationsmittel und technischer Ausrüstung) zu unterstützen.

18. Die Organe der Staatsgewalt der Subjekte der Russländischen Föderation und die Organe der örtlichen Selbstverwaltung sind berechtigt, aus dem Haushalt des jeweiligen Subjekts der Russländischen Föderation oder dem örtlichen Haushalt Mittel zur Unterstützung der Vorbereitung und Durchführung der allrussischen Abstimmung (einschließlich Auszahlungen an Mitglieder der Wahlkommissionen) und zur Bereitstellung von Informationen über die Durchführung der allrussischen Abstimmung an die Bürger der Russländischen Föderation zur Verfügung zu stellen.

19. Zur Vorbereitung und Durchführung der allrussischen Abstimmung können das föderale staatliche Informationssystem „Einheitliches Portal staatlicher und kommunaler Dienstleistungen (Funktionen)", sonstige staatliche Informationssysteme, multifunktionale Zentren zur Erbringung staatlicher und kommunaler Dienstleistungen und (soweit die Voraussetzungen dafür vorhanden sind) elektronische Fernabstimmung eingesetzt werden.

20. Vom Tag der offiziellen Bekanntmachung des Ukaz des Präsidenten der Russländischen Föderation, in dem die allrussische Abstimmung angesetzt wird, bis zum Tag der offiziellen Bekanntmachung der Ergebnisse der allrussischen Abstimmung stellen die durch die Wahlgesetzgebung bestimmten allrussischen und regionalen staatlichen Fernseh- und Rundfunkorganisationen und Redaktionen allrussischer und regionaler staatlicher periodischer Druckerzeugnisse mit wenigstens wöchentlicher Erscheinungsweise der Zentralen Wahlkommission der Russländischen Föderation und den Wahlkommissionen der Subjekte der Russländischen Föderation zum Zweck der Information der Bürger der Russländischen Föderation über die Vorbereitung und Durchführung der allrussischen Abstimmung und der Erläuterung des Verfahrens der Teilnahme an ihr unentgeltlich Sendezeit und Platz in Druckerzeugnissen in folgendem Umfang zur Verfügung:

1) allrussische staatliche Fernseh- und Rundfunkorganisationen stellen wöchentlich mindestens 15 Minuten Sendezeit auf jedem ihrer Kanäle zur Verfügung;

2) regionale staatliche Fernseh- und Rundfunkorganisationen stellen wöchentlich mindestens 10 Minuten Sendezeit auf jedem ihrer Kanäle zur Verfügung;

3) Redaktionen allrussischer staatlicher periodischer Druckerzeugnisse und regionaler staatlicher periodischer Druckerzeugnisse stellen mindestens ein 
Hundertstel des wöchentlichen Umfangs der Druckerzeugnisse zur Verfügung.

21. Die Kosten der Durchführung der allrussischen Abstimmung werden durch die Wahlkommissionen aus Mitteln getragen, die aus dem föderalen Haushalt zur Vorbereitung und Durchführung der allrussischen Abstimmung zur Verfügung gestellt wurden. Die Zentrale Wahlkommission der Russländischen Föderation ist hinsichtlich+ dieser Mittel der Hauptverfügungsberechtigte.

22. Die entscheidungsberechtigten Mitglieder der Wahlkommissionen, die Mitarbeiter der Apparate der Wahlkommissionen und die Mitarbeiter des Föderalen Zentrums für Informatisierung bei der Zentralen Wahlkommission der Russländischen Föderation erhalten nach Maßgabe einer Regelung durch die Zentrale Wahlkommission der Russländischen Föderation eine zusätzliche Arbeitsvergütung (Gratifikation) für die Arbeit zur Vorbereitung und Durchführung der allrussischen Abstimmung.

23. Beträge, die an nicht dauerhaft beschäftigte entscheidungsberechtigte Mitglieder der Wahlkommissionen, an Mitarbeiter des Föderalen Zentrums für Informatisierung bei der Zentralen Wahlkommission und an Bürger, die aufgrund bürgerlichrechtlicher Verträge tätig waren, für unmittelbar mit der Vorbereitung oder Durchführung der allrussischen Abstimmung zusammenhängende Arbeiten oder Dienstleistungen ausgezahlt werden, unterliegen nicht der Einkommensteuer; für sie müssen keine Versicherungsbeiträge abgeführt werden.

24. Das Föderale Gesetz vom 5. April 2013 Nr. 44-FZ „Über das Kontrahierungssystem im Bereich der Beschaffung von Waren, Arbeiten und Dienstleistungen zur Befriedigung staatlichen und kommunalen Bedarfs" ist auf die Beschaffung von Waren, Arbeiten und Dienstleistungen, die mit der Durchführung der allrussischen Abstimmung zusammenhängen, nach Maßgabe der für die Beschaffung von mit der Vorbereitung der Durchführung der allrussischen Abstimmung zusammenhängenden Waren, Arbeiten und Dienstleistungen geltenden Regeln anzuwenden.

25. Die Zentrale Wahlkommission der Russländischen Föderation stellt das Ergebnis der allrussischen Abstimmung nicht später als fünf Tage nach dem Tag der Durchführung der Abstimmung fest.

Die Zahl der Bürger, die an der allrussischen Abstimmung teilgenommen haben, wird anhand der in den Stimmurnen befindlichen Stimmzettel festgestellt.

26. Die Zentrale Wahlkommission der Russländischen Föderation macht das Ergebnis der allrussischen Abstimmung innerhalb von drei Tagen nach der Entscheidung über seine Feststellung offiziell bekannt.

\section{Artikel 3}

1. Dieses Gesetz tritt am Tag seiner offiziellen Bekanntmachung nach der Billigung durch die Organe der gesetzgebenden Gewalt von nicht weniger als zwei Dritteln der Subjekte der Russländischen Föderation in Kraft; ausgenommen sind Artikel 1 und 2 dieses Gesetzes, deren Inkrafttreten besonders geregelt ist. 
2. Nach dem Inkrafttreten dieses Gesetzes legt der Präsident der Russländischen Föderation dem Verfassungsgericht der Russländischen Föderation die Frage vor, ob die nicht in Kraft getretenen Vorschriften dieses Gesetzes mit den Vorschriften der Kapitel 1, 2 und 9 der Verfassung der Russländischen Föderation und die Regelung über das Inkrafttreten von Artikel 1 dieses Gesetzes mit der Verfassung der Russländischen Föderation vereinbar sind.

3. Das Verfassungsgericht der Russländischen Föderation ist verpflichtet, spätestens sieben Tage nach Übermittlung der Frage gemäß Absatz 2 dieses Artikels durch den Präsidenten der Russländischen Föderation ein Gutachten über die Vereinbarkeit (Unvereinbarkeit) der nicht in Kraft getretenen Vorschriften dieses Gesetzes mit den Vorschriften der Kapitel 1, 2 und 9 der Verfassung der Russländischen Föderation und der Regelung des Inkrafttretens von Artikel 1 dieses Gesetzes mit der Verfassung der Russländischen Föderation zu erstatten.

Wird festgestellt, dass die nicht in Kraft getretenen Vorschriften dieses Gesetzes mit Vorschriften der Kapitel 1, 2 und 9 der Verfassung der Russländischen Föderation oder die Regelung zum Inkrafttreten von Artikel 1 dieses Gesetzes mit der Verfassung der Russländischen Föderation unvereinbar sind, treten diese Vorschriften nicht in Kraft und die allrussische Abstimmung wird nicht durchgeführt. Kommt das Verfassungsgericht der Russländischen Föderation in seinem Gutachten zu dem Ergebnis, dass die nicht in Kraft getretenen Vorschriften dieses Gesetzes mit den Vorschriften der Kapitel 1, 2 und 9 der Verfassung der Russländischen Föderation und die Regelung zum Inkrafttreten von Artikel 1 dieses Gesetzes mit der Verfassung der Russländischen Föderation vereinbar sind, wird die allrussische Abstimmung durchgeführt.

4. Artikel 1 dieses Gesetzes tritt (nach Maßgabe der in Absatz 7 dieses Artikels geregelten Besonderheiten) am Tag der offiziellen Bekanntmachung des Ergebnisses der allrussischen Abstimmung in Kraft, wenn die in ihm vorgesehenen Änderungen der Verfassung der Russländischen Föderation durch die allrussische Abstimmung gebilligt worden sind.

5. Die durch Artikel 1 dieses Gesetzes vorgesehenen Änderungen der Verfassung der Russländischen Föderation gelten als gebilligt, wenn mehr als die Hälfte der Bürger der Russländischen Föderation, die an der allrussischen Abstimmung teilgenommen haben, für sie gestimmt haben.

Wenn weniger als die Hälfte der Bürger der Russländischen Föderation, die an der allrussischen Abstimmung teilgenommen haben, für die in Artikel 1 dieses Gesetzes vorgesehenen Änderungen der Verfassung der Russländischen Föderation gestimmt haben, so gelten diese Änderungen als nicht gebilligt und Artikel 1 dieses Gesetzes tritt nicht in Kraft.

6. Die in Artikel 81 Absätze 3 und 3.1 der Verfassung der Russländischen Föderation in der durch dieses Gesetz zur die Änderung der Verfassung der Russländischen Föderation vorgesehenen Fassung enthaltene Regelung über die zulässige Anzahl von Amtszeiten, während derer eine und dieselbe Person das Amt des Präsidenten der Russländischen Föderation innehaben kann, steht einer Kandidatur derjenigen Person, die zum Zeitpunkt des Inkrafttretens dieser Änderungen das Amt des Präsidenten innehatte und (oder) innehat, nach der Aufnahme der genannten Regelung in den Text der Verfassung der Russländischen Föderation 
für die gemäß der Änderung vorgesehene zulässige Zahl von Amtszeiten für das Amt des Präsidenten der Russländischen Föderation nicht entgegen, und zwar unabhängig von der Zahl der Amtszeiten, während derer diese Person dieses Amt zuvor innehatte und (oder) bei Inkrafttreten dieser Änderung innehat.

7. Richter des Verfassungsgerichts der Russländischen Föderation, die dieses Amt am Tag des Inkrafttretens von Artikel 1 dieses Gesetzes innehaben, behalten das Amt eines Richters des Verfassungsgerichts der Russländischen Föderation bis es nach den Vorschriften des Föderalen Verfassungsgesetzes vom 21. Juli 1994 Nr. 1-FKZ „Über das Verfassungsgericht der Russländischen Föderation“ endet. Wenn nach dem Inkrafttreten von Artikel 1 dieses Gesetzes die Zahl der Richter des Verfassungsgerichts der Russländischen Föderation, die am Tag des Inkrafttretens dieses Gesetzes das Amt des Inkrafttretens von Artikel 1 dieses Gesetzes das Amt eines Richters des Verfassungsgerichts der Russländischen Föderation innehaben, der durch Artikel 1 dieses Gesetzes vorgesehenen Zahl entspricht oder sie übersteigt, werden neue Richter des Verfassungsgerichts der Russländischen Föderation nicht ernannt.

8. Nach der Billigung der durch Artikel 1 dieses Gesetzes vorgesehenen Änderungen der Verfassung der Russländischen Föderation im Wege der allrussischen Abstimmung erlässt der Präsident der Russländischen Föderation einen Ukaz über die offizielle Bekanntmachung der geänderten Verfassung der Russländischen Föderation, der auch den Tag des Inkrafttretens der Änderungen angibt. Die offizielle Bekanntmachung der geänderten Verfassung der Russländischen Föderation erfolgt unverzüglich nach der offiziellen Bekanntmachung des Ergebnisses der allrussischen Abstimmung.

Der Präsident der Russländischen Föderation

V. PUTIN

Moskau, Kreml

14. März 2020

Nr. 1-FKZ

Aus dem Russischen von Burkhard Breig 